\title{
Application of the Periodic Average System Model in Dam Deformation Analysis
}

\author{
Yueqian Shen and Teng Huang \\ School of Earth Science and Engineering, Hohai University, No. 1 Xikang Road, Nanjing 210098, China \\ Correspondence should be addressed to Yueqian Shen; syq19881012@126.com
}

Received 4 May 2015; Revised 12 July 2015; Accepted 28 July 2015

Academic Editor: Francisco Alhama

Copyright ( $) 2015$ Y. Shen and T. Huang. This is an open access article distributed under the Creative Commons Attribution License, which permits unrestricted use, distribution, and reproduction in any medium, provided the original work is properly cited.

\begin{abstract}
Dams are among the most important hydraulic engineering facilities used for water supply, flood control, and hydroelectric power. Monitoring of dams is crucial since deformation might have occurred. How to obtain the deformation information and then judge the safe conditions is the key and difficult problem in dam deformation monitoring field. This paper proposes the periodic average system model and creates the concept of "settlement activity" based on the dam deformation issue. Long-term deformation monitoring data is carried out in a pumped-storage power station, this model combined with settlement activity is used to make the single point deformation analysis, and then the whole settlement activity profile is drawn by clustering analysis. Considering the cumulative settlement value of every point, the dam deformation trend is analyzed in an intuitive effect way. The analysis mode of combined single point with multipoints is realized. The results show that the key deformation information of the dam can be easily grasped by the application of the periodic average system model combined with the distribution diagram of settlement activity. And, above all, the ideas of this research provide an effective method for dam deformation analysis.
\end{abstract}

\section{Introduction}

The usage of water resources and operation efficiency of hydraulic engineering facilities are directly affected by the safety, stability, and normal operation of dams. There are safety problems for many dams, because of, for example, hydrology, geology, construction quality, and aging. Therefore, it is necessary to analyze the deformation monitoring data. Afterwards, the running state and deformation law of a dam is adopted. The factors causing the deformation are determined. Then managers can judge the safety of the dam which can provide guiding for the follow-up monitoring work.

Over the past several decades, deformation analysis study for dams has drawn more and more attention, and related research methods are continuously proposed [1-7]. On the basis of the fuzzy clustering theory and method, Wang and Hua analyzed and researched the methods of dam safety monitoring and evaluation [8]. The development of fuzzy mathematics provides a new theoretical basis to solve the problem of deformation analysis with fuzzy language.
Su et al. proposed a method of mining adaptively the pattern between disease and pathogeny of dam to find the genesis on cracks [9]. De Sortis and Paoliani compared a statistical approach with a structural identification technique, and the results showed that the latter method provided a higher degree of accuracy in predicting the future behavior of the structure [10]. Gan et al. introduced viscoelastic constitutive models for concrete materials and bedrocks (a new creep constitutive model for rockfills), and comparative study of numerical calculation results and monitoring data showed good agreement between the computed settlements and measured values [11]. Zhang discussed the feasibility of MF-DFA (Multifractal Detrended Fluctuation Analysis) in determining dam seepage to provide a basis for subsequent modeling calculation [12]. On the basis of the application of artificial neural network principle, Y.-S Kim and B.-T Kim made forecast analysis of relative displacement for a dam [13]. Deng and Wang used improved BP (Back Propagation) neural network model to forecast deformation in dam safety monitoring [14]. The above study enriched and promoted the type of neural network model for analyzing 
dam deformation. Combined with mature theories such as time series analysis techniques, wavelet transformation, and grey theory for dam safety monitoring models and methods made an important contribution to this research.

It is generally known that deformation monitoring of single points cannot coverage an entire dam body. In the light of actual condition, they are just the feature points or layout in a typical section. If the deformation is analyzed using traditional methods which are just single point deformation analysis, the overall analysis of the dam cannot be analyzed intuitively.

The so-called average system, also called moving average, is one of the commonly used tools in analyzing the technical indexes of stock exchange $[15,16]$. Its value lies in the average index of level which is often very important support or resistance. Therefore, it is mostly used by technical analysts. On the basis of the principle of average system, periodic average system model is put forward in this paper. The concept of "settlement activity" is proposed. The "settlement activity" of individual monitoring points is calculated which is regarded as a typical classification index in doing the cluster analysis. Finally, the settlement activity distribution of the whole dam is plotted; the dam deformation is analyzed in a visually way.

\section{Principles of Periodic Average System Model Based on "Settlement Activity"}

2.1. Periodic Average System. The main principle of the periodic average system proposed in this paper is introduced as follows.

(1) Calculating the average of $s_{1}$ and $s_{2}$ periods of abundant continuous data to be analyzed, of which the definition of computation formula is given in

$$
\begin{aligned}
& y_{s_{1}}(k)=\sum_{k=i}^{i+s_{1}-1} \frac{x_{k}}{s_{1}} \\
& y_{s_{2}}(k)=\sum_{k=i}^{i+s_{2}-1} \frac{x_{k}}{s_{2}} \\
& i=k-s_{1}+1 ; k=s_{1}, s_{1}+1, \ldots, n, k=s_{2}, s_{2}+1, \ldots, n,
\end{aligned}
$$

where $y_{s_{1}}$ represents the average of $s_{1}$ periods, $y_{s_{2}}$ represents the average of $s_{2}$ periods, and $k$ is the period.

(2) Calculating the difference between the average value of $s_{1}$ periods and $s_{2}$ periods, according to

$$
\Delta y(k)=y_{s_{1}}(k)-y_{s_{2}}(k) \text {. }
$$

(3) Marking the $s_{1}$ periods and $s_{2}$ periods of periodic average value in the coordinate chart and connecting them by a line, which we call the "periodic average graph."

(4) Combining the periodic average chart with the value of $\Delta y(k)$. Consequently, the trend and deformation of points are analyzed.
Obviously, the theory of periodic average system model is just suitable for deformation analysis of single points. However, it should meet the needs of roughly same monitoring frequency for a large number of consecutive data to be analyzed. And three kinds of situations are shown as follows.

(1) When short periodic average line wears long periodic average line and runs above the long periodic average in this period, the uptrend of the point is shown.

(2) When long periodic average line wears short periodic average line and runs above the short periodic average in this period, the subsidence trend of the point is shown.

(3) When long periodic average line and short periodic average line intertwine together and the trend is in a certain range, the state of relative balance of this point is shown. In this situation, the deformation influenced by factors such as temperature, water level, and rainfall also may be caused by measurement errors.

\subsection{Principle of Periodic Average System Model Based on} "Settlement Activity". In consideration of the weakness of the periodic average system model in the sense that it can analyze a single point, as well as the fact that deformation monitoring points cannot cover the entire of dam body, however, in the light of actual conditions, they are just the feature points or layout in a typical section. Therefore, in order to realize multipoint analysis, how to find the connection between different points should be priority for all, and then deformation of the whole dam body is analyzed. Through overall analysis of the dam, the deformation information of this dam is more intuitive.

The concept of "settlement activity" is proposed based on the periodic average system model. The moving regularity of the whole deformable body in this period to be analyzed is reflected. Then combining the value of "settlement activity" of the individual monitoring point with cluster analysis, the existing monitoring points are classified into several categories. Its main principle is introduced as follows.

$\Delta y(k)_{i}(i=1, \ldots, n)$ of each point is calculated based on the principle of periodic average system model; then the average $\Delta y(k)_{i}(i=1, \ldots, n)$ of all points is calculated. In the light of actual conditions, the critical difference of $\Delta_{0}$ is calculated. "Whether $\Delta y(k)_{i}(i=1, \ldots, n)$ of monitoring points is more than the critical difference $\left(\Delta_{0}\right)$ " performs the evaluation index. "Settlement activity" is defined as the number $(N)$ of cycle average difference more than the evaluation index.

The frequency of deformation monitoring activities for the dam is reflected by "settlement activity." "Settlement activity" of a point is calculated in the following process.

(1) According to the monitoring data to be analyzed and corresponding monitoring cycle $(T)$, the difference $\left(\Delta_{i}\right)$ between the average of $s_{1}$ periods and the average of $s_{2}$ periods in different analyzed cycles is calculated.

(2) According to the difference $\left(\Delta_{i}\right)$ between the average of $s_{1}$ periods and the average of $s_{2}$ periods calculated above, or with the years of experience in observation, a threshold value $\left(\Delta_{0}\right)$ of the difference between different cycles is defined.

(3) The number $(N)$ of the difference $\left(\Delta_{i}\right)$ more than the given threshold value $\left(\Delta_{0}\right)$ is calculated. 
(4) The number of threshold is given; then the scope of the "settlement activity" of different levels is determined. Each level range can be different, and, according to the level of different point, "settlement activity" is calculated.

2.3. Cluster Analysis Based on Settlement Activity. After calculating the "settlement activity" of each monitoring point, taking "settlement activity," average of deformation, and cumulative deformation value as the classification indicators, cluster analysis is adopted for classification. Combining with the accumulated subsidence value, individual abnormal point is excluded. Then, the distribution diagram for the whole dam of "settlement activity" is plotted.

2.4. Advantages of the Theory of Periodic Average System Model Based on "Settlement Activity". In this paper, through periodic average system model, the average of $s_{1}$ cycles $\left(y_{s_{1}}(k)\right)$ and $s_{2}$ cycles $\left(y_{s_{2}}(k)\right)$ is calculated. Then combining $y_{s_{1}}(k)$ with $y_{s_{2}}(k)$, the difference $(\Delta y(k))$ is calculated. $\Delta y(k)$, $y_{s_{1}}(k)$, and $y_{s_{2}}(k)$ ranges are drawn on the same graph; on the basis of $\Delta y(k)$ "settlement activity" is ranked. This approach has several advantages for data analysis, which are shown as follows.

2.4.1. Weaken the Influence of Observation Error. Monitoring result inevitably contains several kinds of errors, especially under poor conditions; it contains a number of errors and gross error. Through calculating $y_{s_{1}}(k)$ and $y_{s_{2}}(k)$, it can effectively weaken the influence of errors on deformation analysis. In the light of actual conditions, the threshold value $\left(\Delta_{0}\right)$ of the difference between different cycles and the width of different levels $\left(\lambda_{i}\right)$ are adjusted appropriately; the influence of errors on adjustment results can be effectively weakened.

Assume that the influence caused by observation conditions on elevation is $\sigma$; that is,

$$
H=\left(H^{\prime} \pm \sigma\right) m m
$$

where $H^{\prime}$ represents the observation elevation and $H$ represents the elevation considering the error of observation conditions.

Assuming that all observation cycles are of equal precision, thus, for the average of $s_{1}$ periods $\left(y_{s_{1}}(k)\right)$,

$$
H_{s_{1}}=\left(H^{\prime} \pm \frac{\sigma}{\sqrt{s_{1}}}\right) m m .
$$

For the average of $s_{2}$ periods $\left(y_{s_{2}}(k)\right)$,

$$
H_{s_{2}}=\left(H^{\prime} \pm \frac{\sigma}{\sqrt{s_{2}}}\right) m m .
$$

Considering the propagation of error law, the influence caused by observation conditions on the difference $(\Delta y(k))$ between different average of $s_{1}$ periods and the average of $s_{2}$ periods is given in

$$
\sigma_{s_{1} s_{2}}=\frac{\sigma}{\sqrt{s_{1}}}+\frac{\sigma}{\sqrt{s_{2}}}=\frac{\sqrt{s_{1}}+\sqrt{s_{2}}}{\sqrt{s_{1}} \cdot \sqrt{s_{2}}} \sigma .
$$

By comparative analysis of (1) and (2), if calculated in the light of conventional analysis, elevation is regarded as research object. We can see clearly that the elevation results always contain the influence of $\sigma$ because of the observation errors. If we are analyzing using the theory of periodic average system model, the influence of observation errors is weakened. From the foregoing discussion, $H_{s_{1}}$ is influenced by the error of $\sigma / \sqrt{s_{1}}, H_{s_{2}}$ is influenced by the error of $\sigma / \sqrt{s_{2}}$, and the difference $(\Delta y(k))$ between different cycles is influenced by the error of $\left(\left(\sqrt{s_{1}}+\sqrt{s_{2}}\right) /\left(\sqrt{s_{1}} \cdot \sqrt{s_{2}}\right)\right) \sigma$. As we can see, along with the increasing of $s_{1}$ and $s_{2}, \sigma / \sqrt{s_{1}}, \sigma / \sqrt{s_{2}}$, and $\left(\left(\sqrt{s_{1}}+\sqrt{s_{2}}\right) /\left(\sqrt{s_{1}} \cdot \sqrt{s_{2}}\right)\right) \sigma$ become smaller. Considering that $s_{1}$ and $s_{2}$ are integers greater than 1 , therefore, $\sigma / \sqrt{s_{1}}$, $\sigma / \sqrt{s_{2}}$, and $\left(\left(\sqrt{s_{1}}+\sqrt{s_{2}}\right) /\left(\sqrt{s_{1}} \cdot \sqrt{s_{2}}\right)\right) \sigma$ are always less than $\sigma$.

2.4.2. Convenient for Global Analysis. The original monitoring data always contains a large number of information. If the deformation hygrographs of all points are drawn in one picture, different deformation hygrograph will be overlapped. Meanwhile, the difference of absolute deformation value between different points is large, so it is difficult to find the regularity of the displacement for the dam, as shown in Figure 1.

\subsubsection{Deformation Information of the Whole Monitoring} Period Is Adopted. Deformation value of monitoring points has always the concern object. However the deformation value is always "static," which can only reflect the deformation information during a certain moment or period. The average of cycles calculated by the periodic average system is continuous, which can reflect the deformation trend during the whole monitoring time, and then the deformation information of the dam is analyzed. Deformation rate is just the average rate during a period of time, which can only reflect the average deformation information during this certain period of time. However, "deformation value" and "deformation rate" cannot reflect the activities of the dam during the whole monitoring time (that is to say the frequency of the displacement cannot be reflected). This problem is approached perfectly by the theory of periodic average system model based on "settlement activity." Then this paper takes some examples to illustrate its advantages.

As shown in Figure 2, deformation hygrographs of monitoring points $\mathrm{A}$ and $\mathrm{B}$ are drawn in red line and blue line, from which we can see that the deformation value of monitoring point $\mathrm{A}$ is greater than monitoring point $\mathrm{B}$ during this period. Taking the "deformation rate" as analysis object, monitoring point $\mathrm{A}$ is greater than monitoring point $\mathrm{B}$ at the time of $t 1$. Meanwhile, for the rest time of this period, the deformation rate of monitoring point $\mathrm{A}$ is less than monitoring point $\mathrm{B}$. By traditional deformation analysis, the analysis results are adopted at a certain time. However, as we can see in Figure 2, although the deformation value of monitoring point $\mathrm{A}$ is always greater than monitoring point $\mathrm{B}$, in terms of deformation activity, monitoring point $\mathrm{B}$ is more active than monitoring point A significantly. If only two monitoring points are analyzed, conclusions can be drawn 


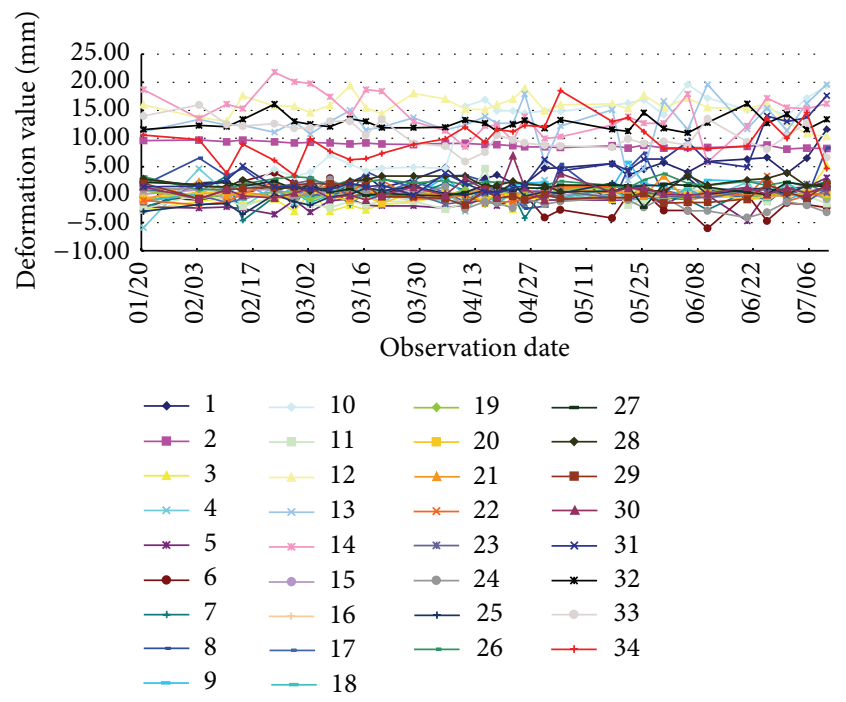

FIGURE 1: Process line about a large number of monitoring points.

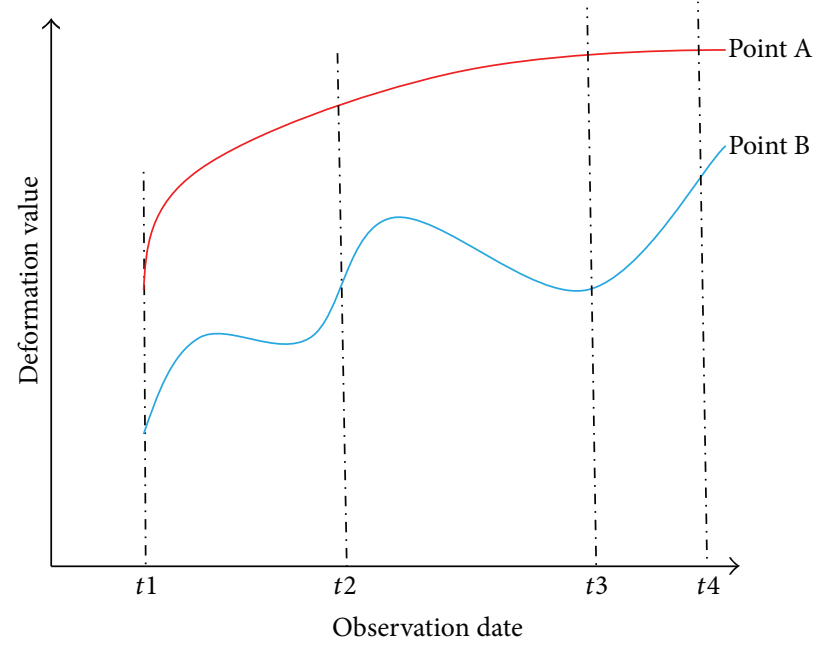

FIGURE 2: Comparison of different monitoring point process line.

through the analysis of deformation hygrograph. As for one deformable body which has dozens of points, the traditional analysis method of drawing deformation hygrograph cannot reflect the deformation activity intuitively in each part of the whole plasmodium. If the theory of periodic average system model based on "settlement activity" is adopted, not only the activity of monitoring point $\mathrm{A}$ and monitoring point $\mathrm{B}$ can be reflected, but also the whole deformation information can be reflected.

\section{Engineering Application and Deformation Analysis}

The dam type of a pumped-storage power station is a reinforced concrete rockfill dam. The elevation of the dam crest is $174 \mathrm{~m}$, the total length of dam crest is $665 \mathrm{~m}$, and upstream and downstream dam slope ratio is $1: 1.4$. The work

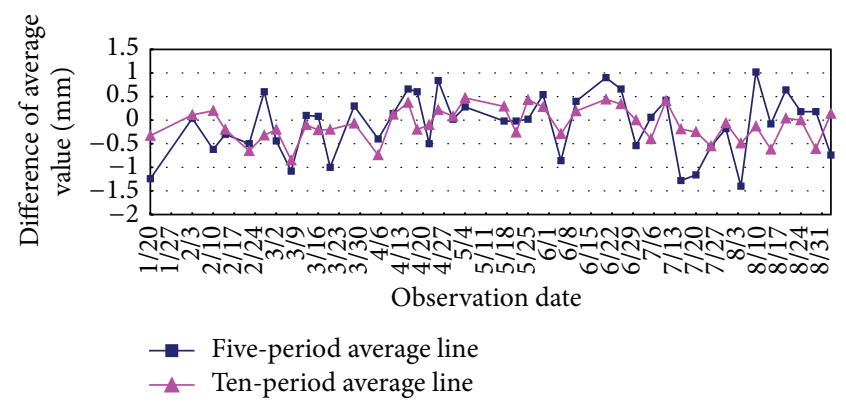

FIgURE 3: Periodic average graph (LD2-3).

of this paper takes years of monitoring data as research objects; monitoring time from January 20, 2008, to August 30,2008 , is selected.

3.1. Application of the Theory of Periodic Average System for Single Point Deformation Analysis. The application of periodic average system in dam deformation analysis for single point has three situations which have been introduced in the above contents. For the value of $s_{1}$ and $s_{2}$, when $s_{1}=1$ and $s_{2}=n(n \geq 2)$, is the extreme case, 1-period average line represents the original observations connecting to line and $n$ periodic average line represents the mean of $n$ periods original observations connecting to line. It is totally meaningless to analyse this extreme case. Here illustrating the application only by the monitoring point LD2-3, in order to analyse intuitively, assuming that $s_{1}=5$ and $s_{2}=10$, periodic average graphs are shown in Figure 3.

In Figure 3, some conclusions can be drawn by analyzing the five-period average line and ten-period average line (where blue line is five-period average line and red line is tenperiod average line).

(1) From January 1 to July 6, five-period average line and ten-period average line are winding together all the way, which show that the monitoring point is in a relatively stable condition during this period.

(2) From July 6 to August 3, five-period average line is below ten-period average line, which shows that the monitoring point has the subsidence trend during this period.

(3) After August 3, we can see that five-period average line wears ten-period average line and it runs above the tenperiod average line to the end of August. Obviously, the monitoring point has a lifting trend during this period.

3.2. Calculation and Analysis of Settlement Activity. According to the above theories, the threshold calculated is $\Delta_{0}=$ $0.6 \mathrm{~mm}$. The times $\left(N_{i}\right)$ more than the given threshold $\left(\Delta_{0}\right)$ of all monitoring points are calculated, respectively, in the period of 100 150 cycles. Then the "settlement activity" is calculated, which is divided into four levels, the specific information is shown in Table 1.

According to the classification criteria of settlement activity, the settlement activity of each point is classified into corresponding level. In order to have access to cluster 


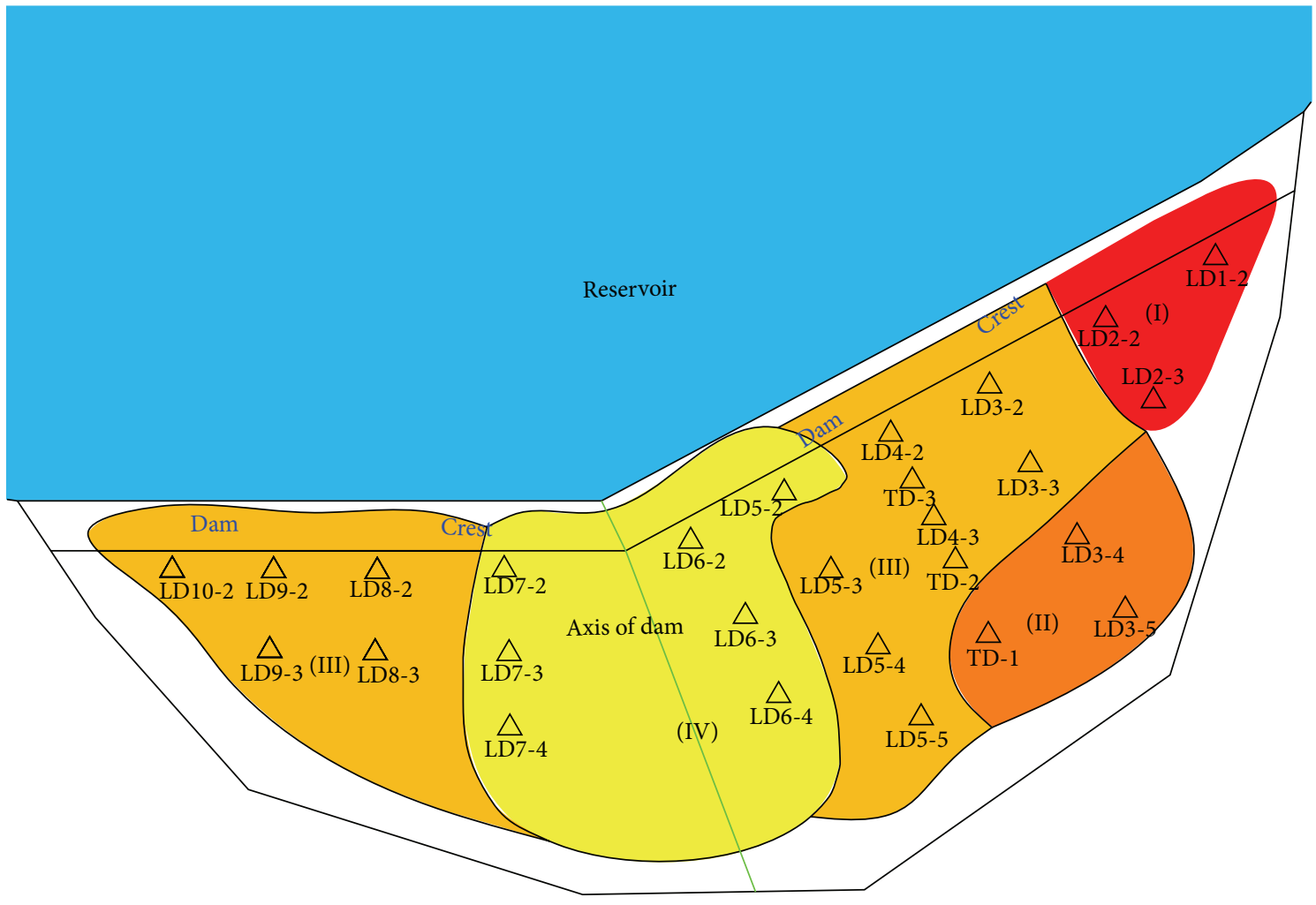

FIGURE 4: Distribution diagram of settlement activity.

TABLE 1: Classification of settlement activity.

\begin{tabular}{lc}
\hline Standard of classification & Settlement activity \\
\hline $0 \leq N \leq 3$ & Inactivity \\
$4 \leq N \leq 6$ & Mild active \\
$7 \leq N \leq 9$ & Moderately active \\
$N \geq 10$ & Highly active \\
\hline
\end{tabular}

analysis, different values are, respectively, given for settlement activity, which is shown in Table 2.

3.3. The Multipoint Overall Analysis for the Dam. According to the classification shown in Table 2, the settlement activity of individual monitoring point, the average of absolute settlement value, and the cumulative settlement are considered to be the classification index. The monitoring points on the dam slope are classified into different categories by cluster analysis (the classified results are shown in Table 3). Then combining categories with the cumulative settlement, individual abnormal points (e.g., TD-3) are removed; finally distribution of settlement activity on dam slope is drawn as shown in Figure 4.

In Figure 4, the sorting for settlement activity of I IV area is I > II > III > IV, so the following information can be shown from Figure 4.

(1) On the dam slope, the most active area of settlement distribution is the right side and downstream of the right.
The settlement activity is relatively weaker on both sides of the area of dam axis of the inflection point.

(2) Comparing the cumulative settlement of all monitoring points, the cumulative settlement of LD6-2, LD5-2, LD42, and LD3-2 is larger than others. But, during the period analyzed, the settlement activity of these points is in the area of relative weakness, which show that the area is more active before the analyzed period but has reached a stable state during the analyzed period.

The settlement activity is decreased significantly.

(3) As we can see from the distribution of settlement activity, from the turning point line of the dam axis to the two sides, the settlement activity is the weakest to increase gradually.

\section{Conclusions}

A large number of dam monitoring data are analyzed. The theory of periodic average system model is put forward based on the average analysis system in stock market. The concept of "settlement activity" is proposed, and then the deformation is analyzed form the perspective of single monitoring point. Afterwards, with the "settlement activity" as the main classification index, all the monitoring points are classified into different categories by the method of cluster analysis; the whole dam settlement activity picture is drawn. The longterm deformation of the dam is analyzed from the perspective of multipoint, which is intuitively shown. What is more, accumulated deformation information is adopted. The ideas 
TABLE 2: Settlement activity of every point.

\begin{tabular}{lccccc}
\hline Point name & Settlement activity & Point name & Settlement activity & Point name & Settlement activity \\
\hline TD-3 & Moderately active (3) & LD7-3 & Inactivity (1) & LD4-2 & Mild active (2) \\
TD-2 & Mild active (2) & LD7-4 & Mild active (2) & LD4-3 & Moderately active (3) \\
TD-1 & Mild active (2) & LD6-2 & Inactivity (1) & LD3-2 & Mild active (2) \\
LD10-2 & Mild active (2) & LD6-3 & Inactivity (1) & LD3-3 & Moderately active (3) \\
LD9-2 & Inactivity (1) & LD6-4 & Inactivity (1) & LD3-4 & Mild active (2) \\
LD9-3 & Mild active (2) & LD5-2 & Inactivity (1) & LD3-5 & Mild active (2) \\
LD8-2 & Mild active (2) & LD5-3 & Moderately active (3) & LD2-2 & Highly active (4) \\
LD8-3 & Mild active (2) & LD5-4 & Moderately active (3) & LD2-3 & Highly active (4) \\
LD7-2 & Mild active (2) & LD5-5 & Moderately active (3) & LD2-1 & Highly active (4) \\
\hline
\end{tabular}

TABLE 3: Classification results of cluster analysis.

\begin{tabular}{ll}
\hline Category & Point name \\
\hline I & LD1-2, LD2-2, LD2-3, TD-3 \\
II & TD-1, LD3-4, LD3-5 \\
III & LD3-2, LD3-3, LD4-2, LD4-3, TD-2, LD5-3, LD5-4, \\
& LD5-5, LD10-2, LD9-2, LD8-2, LD9-3, LD8-3 \\
IV & LD7-2, LD7-3, LD7-4, LD6-2, LD5-2, LD6-3, LD6-4 \\
\hline
\end{tabular}

and methods of this paper provide an effective method for dam deformation analysis. Meanwhile, the results provide practical guidance for managers to judge the safety situation of dams. However, in the process of deformation analysis, several problems remain to be improved as follows.

(1) Combining the theory of periodic average system model with corresponding charts, the deformation trend of the monitoring points is easily adopted. Therefore, it can be regarded as a kind of simple and practical method for a certain trend analysis and forecasting of monitoring points; however, it is unable to predict the concrete value of the deformation, which should be combined with other mathematical models.

(2) The deformation information is reflected clearly by the distribution diagram of settlement activity based on cluster analysis, but it cannot quantify the value of deformation, as well as the measurement error; environmental information is not considered. It will be the focus of next research.

\section{Conflict of Interests}

The authors declare that there is no conflict of interests regarding the publication of this paper.

\section{Acknowledgments}

This work has been partially supported by the Surveying and Mapping Project in Jiangsu province (JSCHKY201301) and the College Postgraduate Research and Innovation project in Jiangsu province (CXZZ13_0264).

\section{References}

[1] O. CrePon and M. Lino, "An analytical approach to monitoring," International Water Power and Dam Construction, vol. 51, no. 6, pp. 52-54, 1999.

[2] E. C. Kalkani, "Polynomial regression to forecast earth dam piezometer levels," Journal of Irrigation \& Drainage Engineering, vol. 115 , no. 4 , pp. $545-555,1989$.

[3] J. W. Kantelhardt, S. A. Zschiegner, E. Koscielny-Bunde, S. Havlin, A. Bunde, and H. E. Stanley, "Multifractal detrended fluctuation analysis of nonstationary time series," Physica A: Statistical Mechanics and its Applications, vol. 316, no. 1-4, pp. 87-114, 2002.

[4] J. K. Lou and L. Yan, "Seismic upgrade of coquitlam dam," Chinese Journal of Geotechnical Engineering, vol. 29, no. 11, pp. 1669-1678, 2008 (Chinese).

[5] F. Alcrudo and J. Mulet, "Description of the Tous Dam break case study (Spain)," Journal of Hydraulic Research, vol. 45, pp. 45-57, 2007.

[6] D. Vjushin, R. B. Govindan, R. A. Monetti, S. Havlin, and A. Bunde, "Scaling analysis of trends using DFA," Physica A, vol. 302, no. 1-4, pp. 234-243, 2001.

[7] L. E. Chouinard, B. Ahmadi Nedushan, N. Feknous et al., "Statistical analysis in real time of monitoring data for idukki arch dam," in Proceedings of the 2nd International Conference on Dam Safety Evaluation, pp. 381-385, Trivandrum, India, 1996.

[8] T. S. Wang and X. S. Hua, "Building of monitoring model for dam safety based on fuzzy cluster algorithm," Journal of Hydraulic Engineering, no. 6, pp. 115-118, 2003 (Chinese).

[9] H.-Z. Su, Z.-P. Wen, and H.-C. Dai, "A method of mining adaptively the pattern between disease and pathogeny of dam," in Proceedings of the International Conference on Machine Learning and Cybernetics, vol. 5, pp. 3050-3055, November 2003.

[10] A. De Sortis and P. Paoliani, "Statistical analysis and structural identification in concrete dam monitoring," Engineering Structures, vol. 29, no. 1, pp. 110-120, 2007.

[11] L. Gan, Z.-Z. Shen, and L.-Q. Xu, "Long-term deformation analysis of the jiudianxia concrete-faced rockfill dam," Arabian Journal for Science and Engineering, vol. 39, no. 3, pp. 1589-1598, 2014.

[12] S. P. Zhang, "The use of MF-DFA based on multi-fractal theory in the safety-monitoring of dam seepage," Applied Mechanics \& Materials, vol. 448-453, pp. 1072-1075, 2014. 
[13] Y.-S. Kim and B.-T. Kim, "Prediction of relative crest settlement of concrete-faced rockfill dams analyzed using an artificial neural network model," Computers and Geotechnics, vol. 35, no. 3, pp. 313-322, 2008.

[14] X. S. Deng and X. Z. Wang, "Neural network method for forecasting dam deformation based on historical displacement," Hydropower Automation and Dam Monitoring, vol. 18, no. 2, pp. 51-53, 2004 (Chinese).

[15] E. F. Fama, "Efficient capital markets: II," The Journal of Finance, vol. 46, no. 5, pp. 1575-1617, 1991.

[16] W. Brock, J. Lakonishok, and B. Lebaron, "Simple technical trading rules and the stochastic properties of stock returns," The Journal of Finance, vol. 47, no. 5, pp. 1731-1764, 1992. 


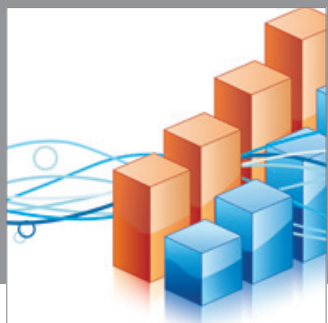

Advances in

Operations Research

mansans

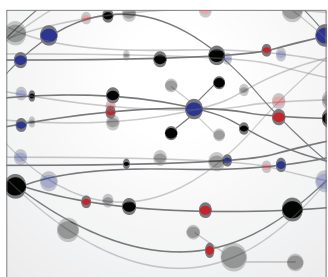

The Scientific World Journal
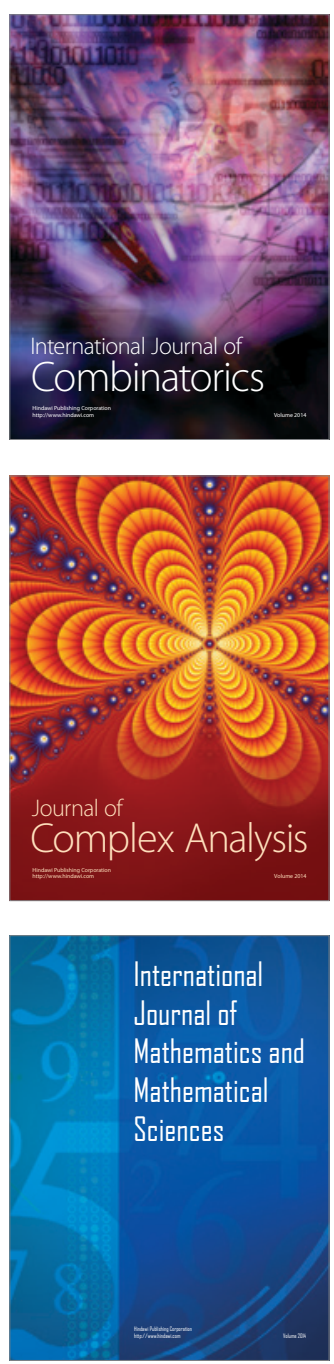
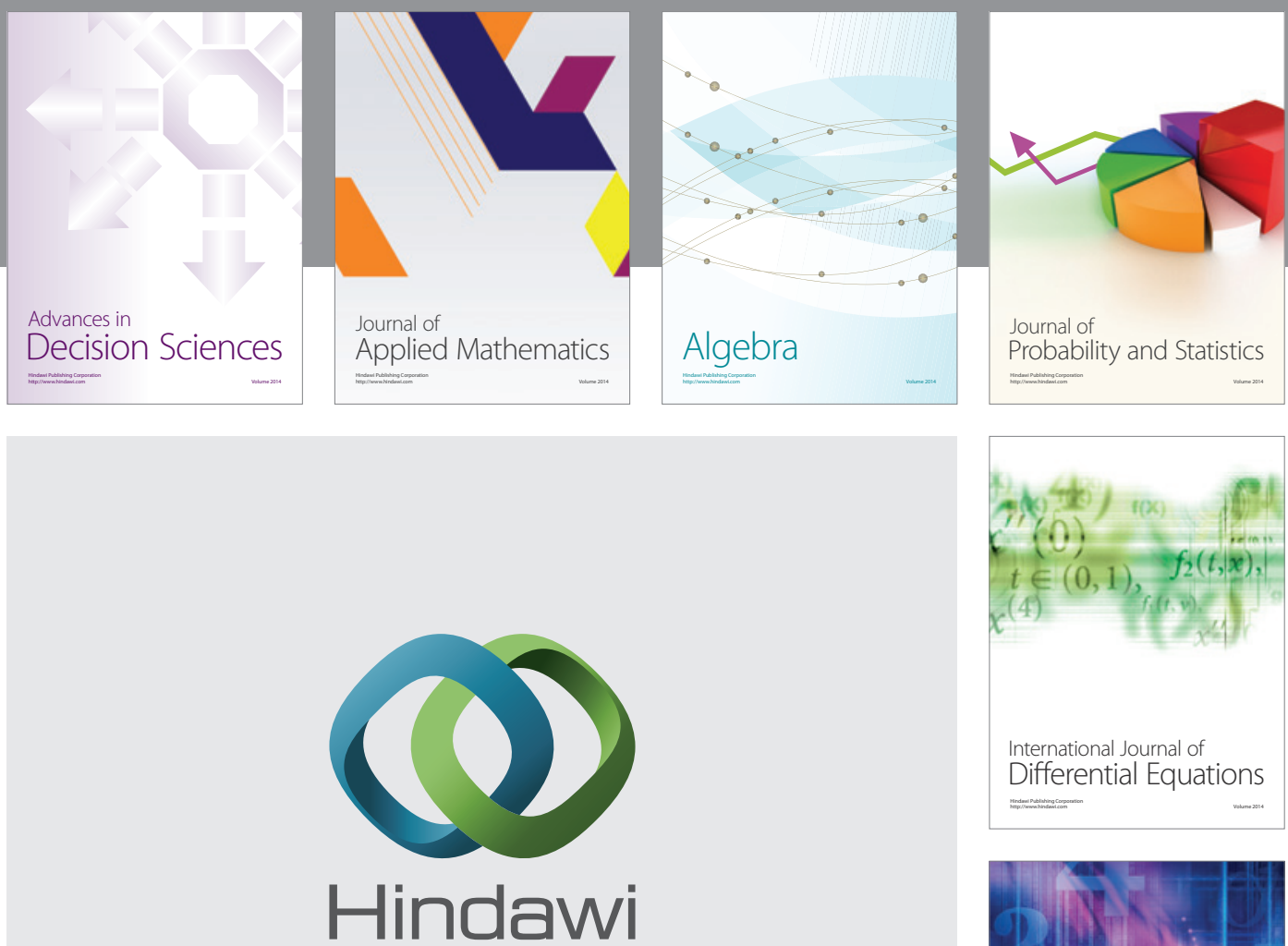

Submit your manuscripts at http://www.hindawi.com
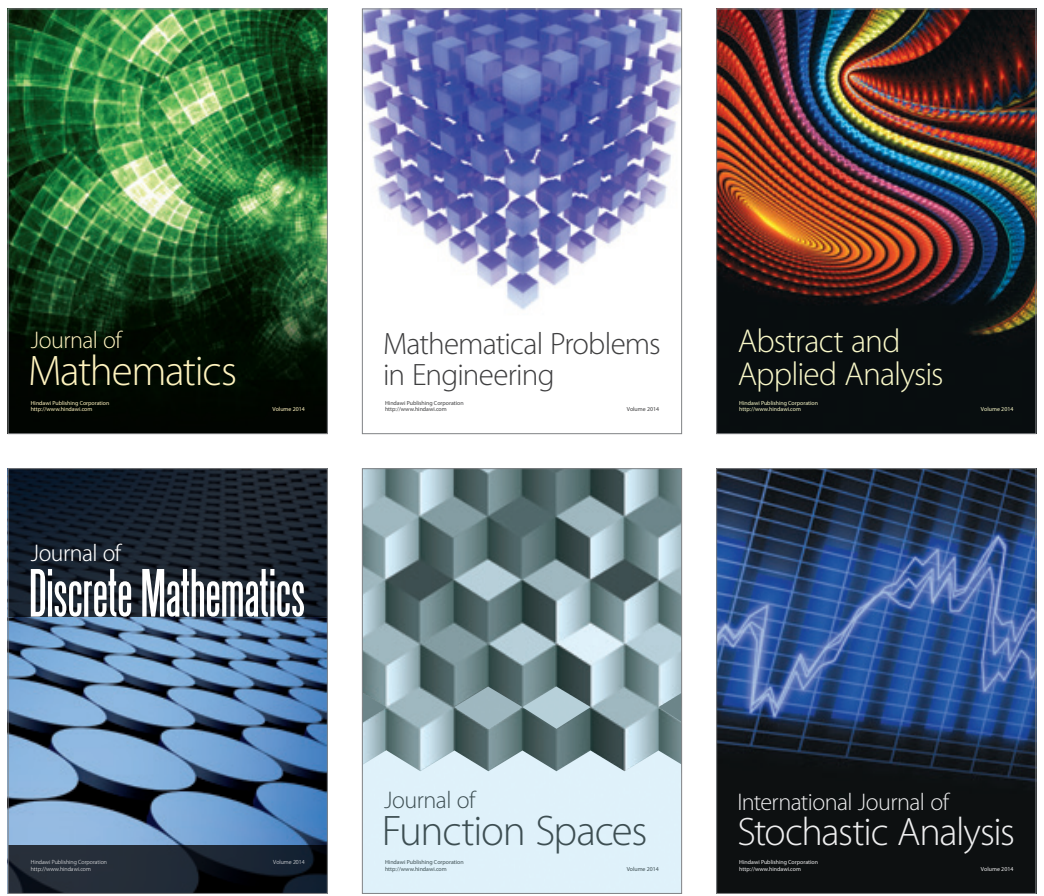

Journal of

Function Spaces

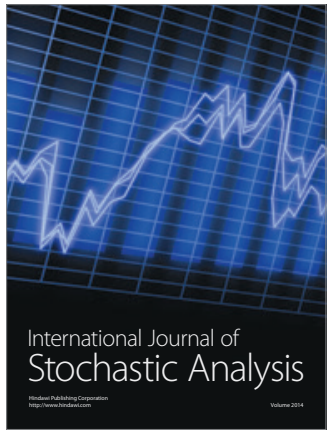

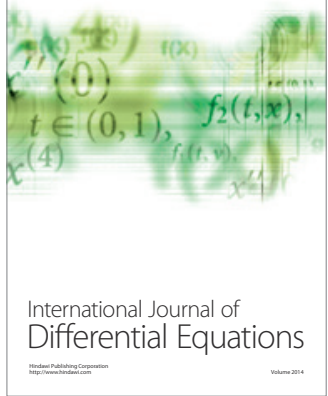
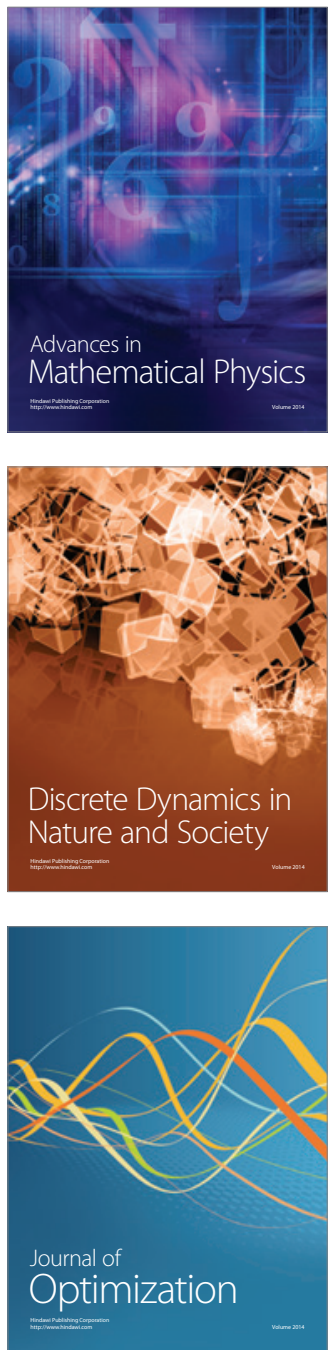